\title{
Surgical correction for Tessier number 7 craniofacial cleft using a medially overcorrected design
}

\author{
Jeong Yeop Ryu, Pil Seon Eo, Lulu Tian, Joon Seok Lee, Jeong Woo Lee, Kang Young Choi, \\ Jung Dug Yang, Ho Yun Chung, Byung Chae Cho \\ Department of Plastic and Reconstructive Surgery, School of Medicine, Kyungpook National University, Daegu, Korea
}

Background Various surgical techniques have been used to correct Tessier number 7 craniofacial cleft, which involves macrostomia, ear deformity, and hemifacial microsomia. To achieve symmetrical and satisfactory results in patients with macrostomia, the authors performed a 1-mm medial overcorrection on the cleft side and evaluated the results of this procedure.

Methods A retrospective medical record review of patients diagnosed with Tessier number 7 craniofacial cleft from March 1999 to February 2017 was performed. Using clinical photographs, outpatient clinic records, and operative records, information was recorded regarding concurrent congenital anomalies, postoperative complications, and follow-up. Using Photoshop CS2, the length of both sides of the lip was compared. The ratio of these lengths was calculated to evaluate lip symmetry.

Results Of the patients treated at the Department of Plastic and Reconstructive Surgery at Kyungpook National University Chilgok Hospital, 11 (male-to-female sex ratio, 7:4) were diagnosed with Tessier number 7 craniofacial cleft. Concurrent congenital anomalies included skin tag, hemifacial microsomia, and cleft palate. The mean duration of follow-up was $78.273 \pm 72.219$ months and the mean ratio of the lengths of both sides of the lip was $1.048 \pm 0.071$. Scar widening occurred as a postoperative complication in some patients. No cases of wound infection, bleeding, or wound dehiscence occurred.

Conclusions For the successful correction of macrostomia, plastic surgeons should consider both functional and aesthetic problems of the lip. Adequate repair of the orbicularis oris muscle, skin closure with Z-plasty, and medial overcorrection of the neo-oral commissure led to good results in our patients.

Keywords Orofacial cleft 7 / Macrostomia / Surgical procedures, operative

Received: 11 Oct 2018 • Revised: 28 Dec 2018 - Accepted: 9 Jan 2019

pISSN: 2234-6163 • elSSN: 2234-6171 • https://doi.org/10.5999/aps.2018.01193・ Arch Plast Surg 2019;46:16-22
Correspondence: Byung Chae Cho Department of Plastic and Reconstructive Surgery, School of Medicine, Kyungpook National University, 130 Dongdeok-ro, Junggu, Daegu 41944, Korea Tel: +82-53-420-5691

Fax: +82-53-425-3879

E-mail: bccho@knu.ac.kr

\section{INTRODUCTION}

Tessier number 7 craniofacial clefts, also referred to as temporozygomatic facial clefts or transverse facial clefts, are the most lat- erally-located subtype of craniofacial clefts. They are a rare congenital anomaly, with a frequency of $1 / 60,000$ to $1 / 300,000$ normal births [1-4], and are characterized by soft tissue abnormalities, such as macrostomia, external ear deformity, and hemi- 
facial microsomia. Among these abnormalities, macrostomia is defined as widening of the mouth at the oral commissure, and involves inferolateral transposition of the oral commissure and atrophy of the orbicularis oris muscle. Considering the importance of the lips, macrostomia presents both aesthetic and functional problems, such as oral incompetence and difficulties in facial expression, mastication, and speech. The treatment of choice for macrostomia is surgical correction. The most important aspects of surgical correction include properly repairing the orbicularis oris muscle to restore function, forming a new oral commissure that is symmetric, and ensuring adequate skin and oral mucosal closure in the extended cleft.

However, the neo-oral commissure created by oral commissuroplasty may experience relapse in the inferolateral direction due to scar contracture, which is a frequently-occurring phenomenon in which a postoperative scar causes contraction of the surrounding soft tissue. Therefore, it is important to anticipate this possibility in advance and to take steps to minimize it. To prevent such cases of relapse, we designed a surgical procedure for patients with macrostomia, in which overcorrection (medially, by approximately $1 \mathrm{~mm}$ ) of the neo-oral commissure on the cleft side was performed together with Z-plasty. This paper presents a discussion of this surgical procedure and an analysis of its outcomes.

\section{METHODS}

\section{Patients and evaluation}

The present retrospective study included all patients diagnosed with Tessier number 7 cleft who underwent surgery for macrostomia at the Department of Plastic and Reconstructive Surgery of Kyungpook National University Chilgok Hospital between March 1999 and February 2017. We classified patients according to whether they had isolated macrostomia or macrostomia with accompanying deformities, such as external ear deformity or hemifacial microsomia, and analyzed their clinical photographs and medical records in order to assess postoperative complications. To evaluate the symmetry of the lips, a single evaluator measured the lengths of both sides of the lips using Photoshop CS2 (Adobe Inc, San Jose, CA, USA) and calculated the ratio of these lengths. The distance from the peak of Cupid's bow to the oral commissure on the cleft side was defined as Dc, and the distance on the normal side was defined as Dn. The ratio of Dc to Dn was calculated as follows: distance ratio $=\mathrm{Dc} / \mathrm{Dn}$.

\section{Surgical technique}

The patients were placed in the supine position, and general anesthesia was induced through an endotracheal tube. To facilitate symmetry of the lips, the tube was placed on the midline of the lower lip. The neck was placed in a neutral position, and $5 \%$ povidone iodine solution and normal saline were used to drape the site cleanly. A marking pen was used to mark the oral commissure on the normal side (point A) and on the affected side (point B). Both peaks of Cupid's bow (points C1 and C2) were marked along the vermilion border of the upper lip, while the midpoint of the lower lip (point $\mathrm{Lm}$ ) was also marked along the vermilion border. In designing the neo-oral commissure on the affected side for the upper lip, the distance between the oral commissure and the peak of Cupid's bow on the normal side (A to C1) was measured, and the point of symmetry from the peak of Cupid's bow on the affected side (point C2) was marked (point B'). Considering the possibility of relapse due to postoperative scar contracture, the actual neo-oral commissure was designed to be at a point 1 $\mathrm{mm}$ medial from the point of symmetry. For the lower lip, the distance between the midpoint of the normal side and oral commissure ( $\mathrm{Lm}$ to $\mathrm{A}$ ) was measured, and the point of symmetry on the affected side (point $\mathrm{B}^{\prime \prime}$ ) was designated. Again considering the possibility of relapse, the actual neo-oral commissure was again designed to be at a point $1 \mathrm{~mm}$ medial from the point of symmetry. A vertical incision line was designated from the points marked as the neo-oral commissure in the upper and lower lips (points $\mathrm{B}^{\prime}$ and $\mathrm{B}^{\prime \prime}$ ) to the buccal mucosa, and the tissue cut along the vermilion border was designed (Figs. 1, 2). After injecting 2\% lidocaine solution mixed with 1:100,000 epinephrine along the incision line, a number 15 blade was used to make the incision along the incision line. After dissecting the skin and the orbicularis oris muscle, the buccal mucosa on the cleft side was repaired. To repair the muscle, the orbicularis oris muscle on the cleft side was overlapped by approximately $5 \mathrm{~mm}$ and repaired using 4-0 Vicryl for firm postoperative muscle adhesion (Fig. 3). If the belly of the zygomaticus major, risorius, and depressor anguli oris muscles were mis-oriented due to the cleft, the neo-modiolus was reconstructed by re-orienting these muscles. After repairing the orbicularis oris muscle, Z-plasty was planned for skin closure to minimize scar contracture on the skin. The direction of the upper limb in the Z-plasty was designed to be as parallel as possible to the nasolabial fold, while the direction of the lower limb was designed to be parallel to the marionette line. The remaining skin incision site was sutured in a layer-by-layer manner, and the procedure was completed after applying foam dressing.

\section{RESULTS}

A total of 11 patients with Tessier number 7 cleft were admitted to the Department of Plastic and Reconstructive Surgery at our hospital between March 1999 and February 2017 (male-to-fe- 


\section{Fig. 1. Schematic images for correction of macrostomia}

(A) In the preoperative design, medial overcorrection on the cleft-side was designed. (B) For firm adhesion of orbicularis oris muscle, myoplasty with overlapping technique was performed. (C) Skin closure was conducted with Z-plasty. A, oral commissure on the normal side; B, oral commissure on the cleft side; C1, peak of Cupid's bow on the normal side; C2, peak of Cupid's bow on the cleft side; B', symmetrical point of upper lip which will be defined to neo-oral commissure on the cleft side compared with the normal; B", symmetrical point of lower lip on the cleft side compared with the normal; D', $1 \mathrm{~mm}$ medial from the point B', which will be defined to actual neo-oral commissure; D", $1 \mathrm{~mm}$ medial from the point B", Lm, midpoint of the lower lip.

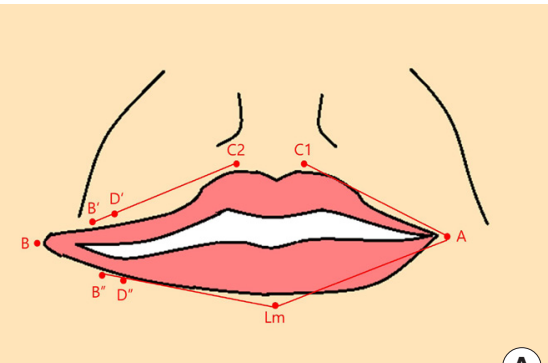

(A)
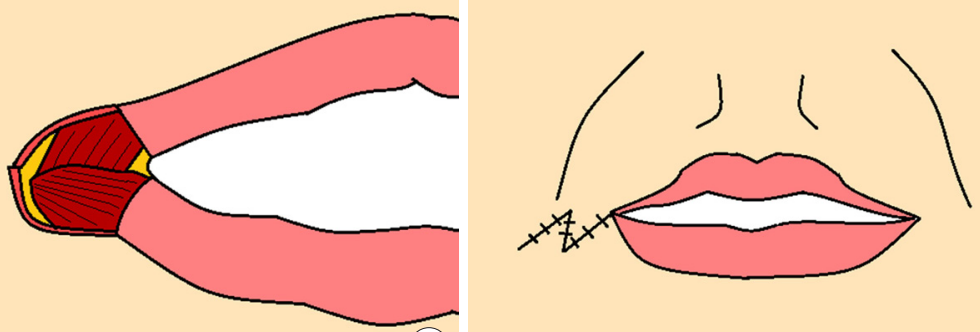

(B)

(C)

\section{Fig. 2. Preoperative design for macrostomia correction}

$A$, oral commissure on the normal side; $B$, oral commissure on the cleft side; $C 1$, peak of Cupid's bow on the normal side; $\mathrm{C}_{2}$, peak of Cupid's bow on the cleft side; B', symmetrical point of upper lip which will be defined to neo-oral commissure on the cleft side compared with the normal; B", symmetrical point of lower lip on the cleft side compared with the normal; Lm, midpoint of the lower lip.

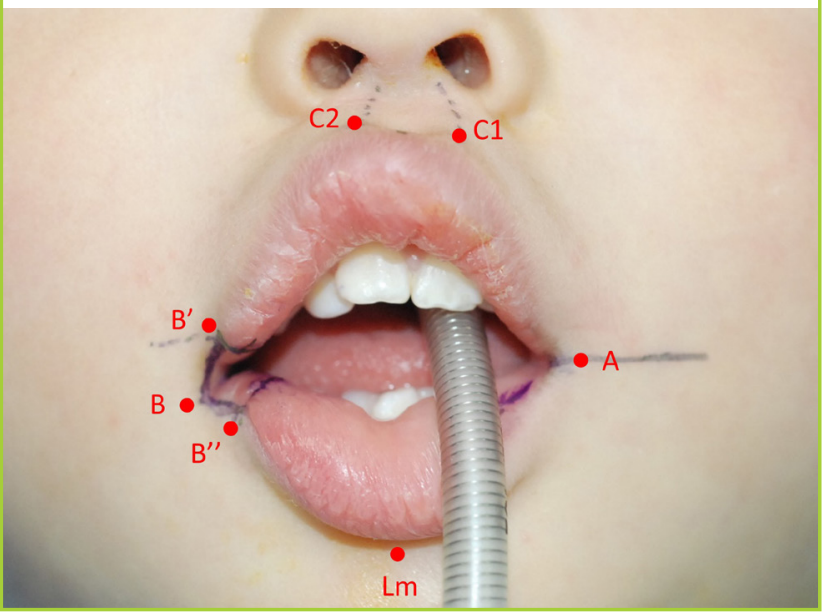

male sex ratio, 7:4). Eight patients had a lesion on the right side and three had a lesion on the left side. Three patients had isolated macrostomia with no other lesions, four had macrostomia with skin tag, two had macrostomia with hemifacial microsomia, and two had macrostomia with hemifacial microsomia and skin tag. The mean follow-up period was $78.273 \pm 72.219$ months. The mean ratio of the lengths of both sides of the lips was calculated as $1.048 \pm 0.071 \mathrm{in}$ an analysis of postoperative photos using Photoshop CS2 (Table 1). With respect to complications, scar widening occurred in four patients, requiring subsequent secondary scar revision. Other complications, such as postoperative infection, bleeding, and wound dehiscence, did not occur.

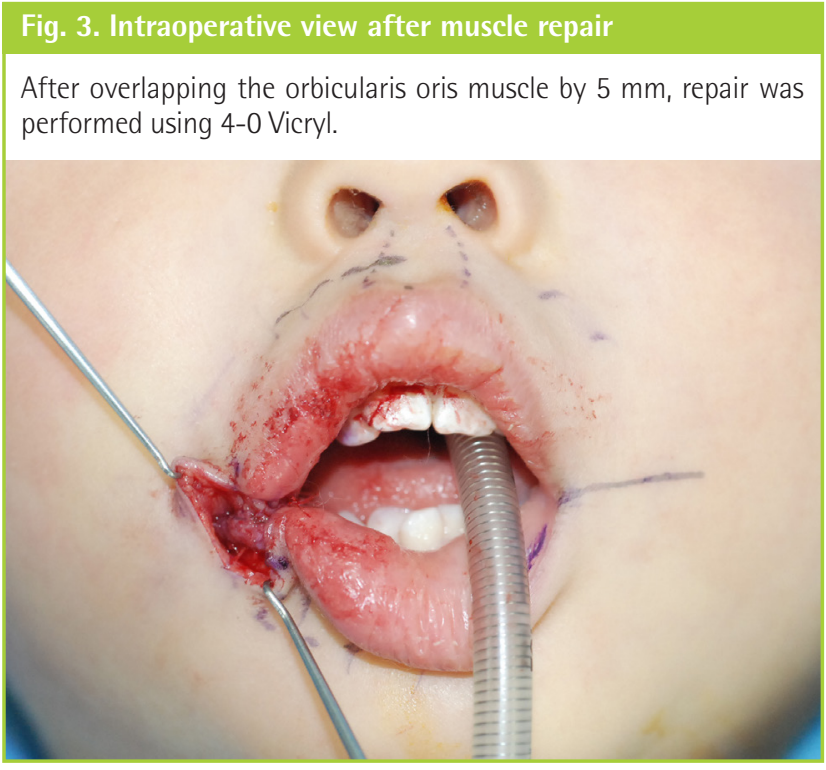

\section{Case 1}

A 4-month-old girl was admitted with chief complaints of right macrostomia and preauricular skin tag. She was diagnosed with right Tessier number 7 craniofacial cleft, and excision and primary closure were performed for the right preauricular skin tag. The procedure for macrostomia was designed with a 1-mm medial overcorrection relative to the normal side, and the correction was performed via Z-plasty during skin closure (Fig. 4).

\section{Case 2}

A 9-month-old girl with right Tessier number 7 craniofacial cleft was admitted with chief complaints of preauricular skin tag and macrostomia. Excision and primary closure were performed for the preauricular skin tag. The procedure for macrostomia was designed with a 1-mm medial overcorrection relative to the nor- 


\section{Fig. 4. A 4-month-girl with macrostomia}

(A) Macrostomia on the right side was seen. (B) The actual neo-oral commissure was designed to be at a point $1 \mathrm{~mm}$ medial from the point of symmetry. (C) Skin closure with Zplasty was performed. (D) At the time of 2 years after correction, symmetry of lip was maintained well.
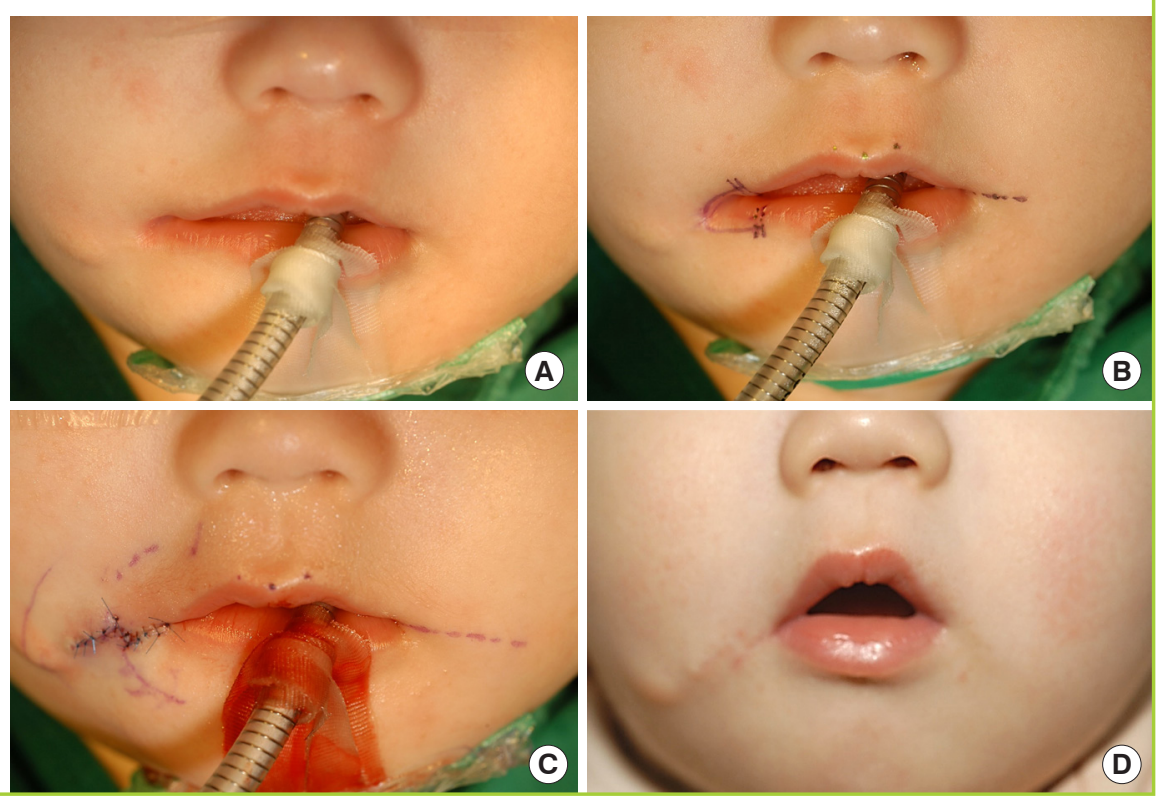

Table 1. Clinical information of the Tessier number $7 \mathrm{cleft}$ patients

\begin{tabular}{|cllcll|}
\hline $\begin{array}{c}\text { Patient } \\
\text { no. }\end{array}$ & Sex & Site & $\begin{array}{c}\text { Duration } \\
\text { of } \\
\text { follow- } \\
\text { up (mo) }\end{array}$ & Concurrent anomaly & $\begin{array}{c}\text { Dc/Dn } \\
\text { ratio }\end{array}$ \\
\hline 1 & Male & Right & 12 & Hemifacial microsomia, skin tag & 1.061 \\
2 & Male & Left & 168 & Hemifacial microsomia & 0.958 \\
3 & Female & Left & 26 & None & 1.111 \\
4 & Male & Left & 156 & None & 1.149 \\
5 & Female & Right & 12 & Skin tag & 1.140 \\
6 & Male & Right & 24 & Skin tag & 0.995 \\
7 & Male & Right & 91 & Hemifacial microsomia & 0.944 \\
8 & Female & Right & 168 & Skin tag & 1.014 \\
9 & Male & Right & 24 & Skin tag & 1.089 \\
10 & Female & Right & 168 & None & 1.041 \\
11 & Male & Right & 12 & Hemifacial microsomia, skin tag & 1.000 \\
\hline
\end{tabular}

Dc, distance from the peak of Cupid's bow to the oral commissure on the cleft side; Dn, distance from the peak of Cupid's bow to the oral commissure on the normal side.

mal side, and the correction was performed via Z-plasty during skin closure. During 14 years of follow-up after the procedure, the right neo-oral commissure that was overcorrected medially by $1 \mathrm{~mm}$ migrated and achieved symmetry with the normal side. The Z-plasty scar had become faint, making it less conspicuous in comparison to the surrounding tissues (Fig. 5).

\section{Case 3}

A 4-month-old boy who had been diagnosed with left Tessier number 7 craniofacial cleft was admitted with chief complaints of preauricular skin tag and macrostomia. Excision and primary closure were performed for the preauricular skin tag. The procedure for macrostomia was designed with a 1-mm medial overcorrection relative to the normal side, and the correction was performed via Z-plasty during skin closure. During 14 years of follow-up after the procedure, the left neo-oral commissure that was overcorrected medially by $1 \mathrm{~mm}$ migrated and achieved symmetry with the normal side. The skin closure scar on the left side was not prominent (Fig. 6).

\section{DISCUSSION}

Craniofacial clefts manifest with a variety of clinical features, and many attempts have been made to classify them [5]. Tessier's classification, which is still widely used, categorizes craniofacial clefts using a numbering system that ranges between 0 and 14 [6]. Tessier number 7 craniofacial cleft, which is the most common type of craniofacial cleft, is also referred to as temporozygomatic facial cleft, craniofacial macrosomia, hemifacial macrosomia, otomandibular dysostosis, first and second branchial arch syndrome, auriculobranchiogenic dysplasia, hemignathia and microtia syndrome, oroaural cleft (American Association of Cleft Palate Rehabilitation, AACPR), group B1 lateral otocephalic branchigenic deformity, and zygotemporal dysplasia. This lateral craniofacial cleft mostly has a horizontal orientation or advances obliquely towards the ipsilateral ear, and reports have indicated that it does not invade beyond the anterior border of the masseter muscle in most cases [5].

The development of the face takes place between the 5th and 8 th weeks through migration and cell differentiation of the first 


\section{Fig 5. A 9-month-girl with macrostomia}

(A) The actual neo-oral commissure was designed to be at a point $1 \mathrm{~mm}$ medial from the point of symmetry (point A'). (B) At the time of 14 years after correction, symmetry of lip was maintained well. (C) Postoperative length of the cleft-side lip was measured to $24 \mathrm{~mm}$ (peak of Cupid's bow to oral commissure). (D) Length of the normal-side lip was measured to $24 \mathrm{~mm}$ (peak of Cupid's bow to oral commissure).
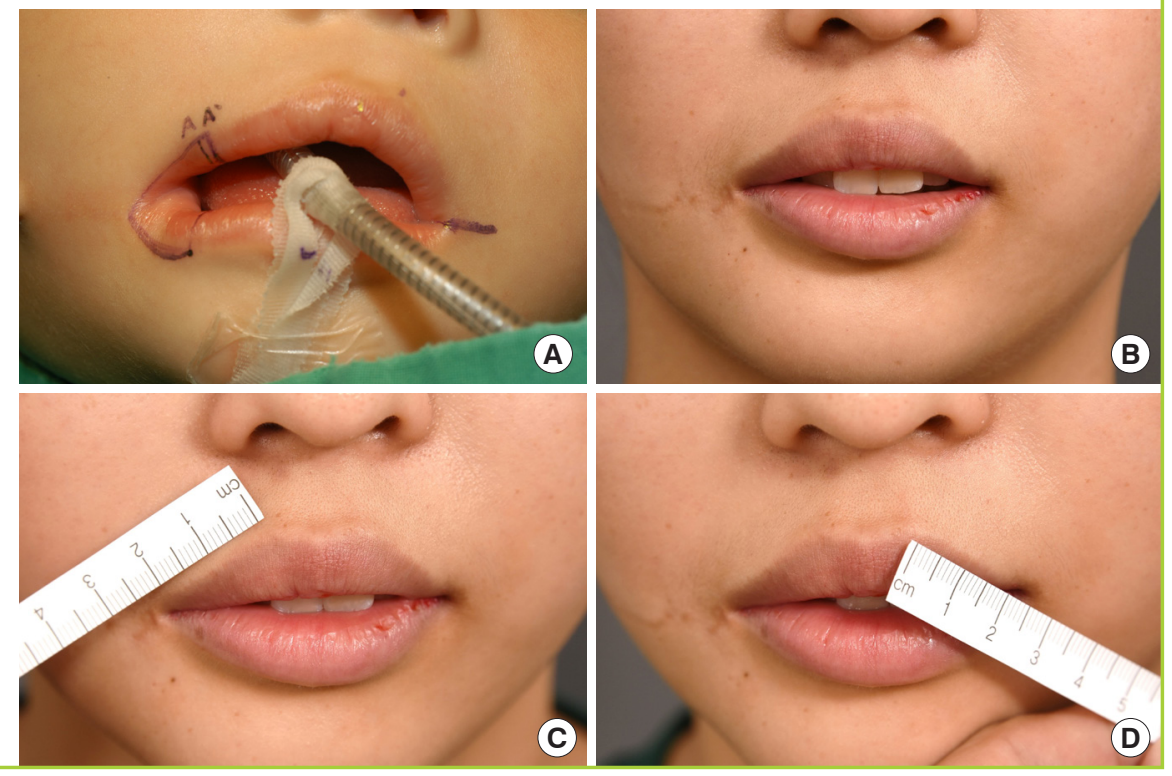

Fig. 6. A 4-month-boy with macrostomia

(A) Macrostomia on the left side was seen. (B) At the time of 14 years after correction, symmetry of lip was maintained well.

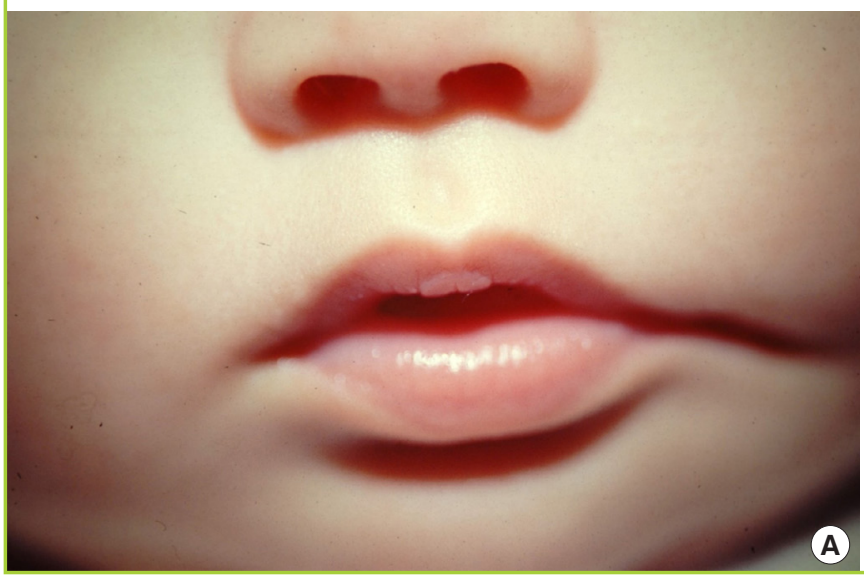

and second branchial arches. The first branchial arch undergoes remodeling via maxillary swelling and caudal mandibular swelling; the maxillary swelling develops into the maxillary prominence and the mandibular swelling into the mandibular prominence. Normally, the maxillary and mandibular prominences meet at the orotragal line and fuse together to obliterate the orotragal line during the 4 th or 5th week [7-9]. However, if this fusion process is impaired or does not take place, macrostomia may appear.

Macrostomia may appear by itself or together with other deformities. Accompanying deformities include hemifacial macrosomia and external ear deformity, the latter of which may appear in various forms, ranging from preauricular skin tag to anotia [5]. Bone anomalies include mandibular hypoplasia, maxillary

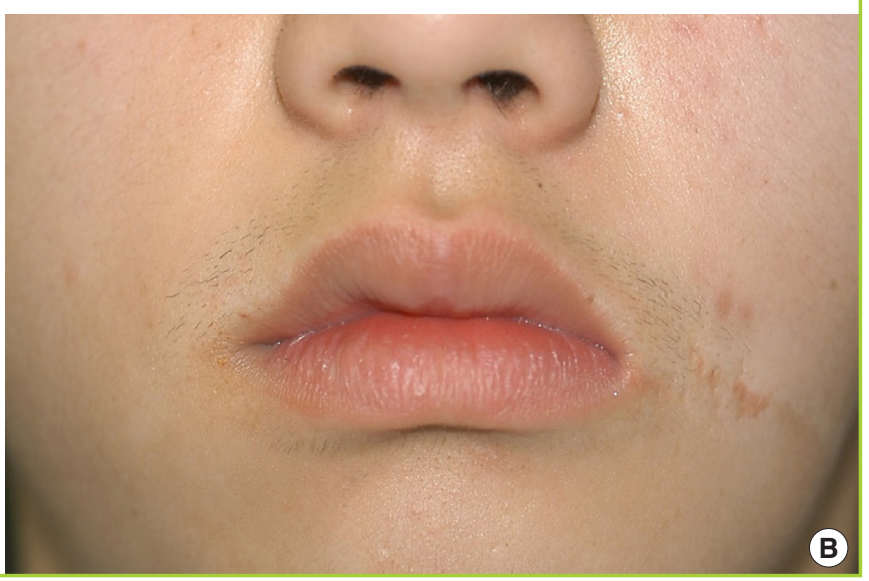

duplication, and zygomatic bone anomalies. Although statistical significance could not be determined owing to the small sample size, the present study found isolated macrostomia in five patients and macrostomia with skin tag in seven patients, indicating that a high percentage of patients were affected with macrostomia accompanied with skin tag. Moreover, two patients had accompanying hemifacial microsomia and three patients had both skin tag and hemifacial microsomia (Fig. 2).

Macrostomia presents both aesthetic and functional problems. Aesthetic problems include an asymmetrical lip contour relative to that on the normal side, while functional problems include feeding difficulties, drooling, and incoherent speech [10]. Therefore, the surgical goals for macrostomia are to create lips that are symmetrical to those on the normal side and to pro- 
mote normal lip function by properly repairing the orbicularis oris muscle. To achieve these goals, the surgical procedure for macrostomia should include myoplasty of the orbicularis oris muscle, commissuroplasty, and skin and buccal mucosal closure on the cleft [7].

Regarding myoplasty of the orbicularis oris muscle, the repaired free ends of the muscle should be positioned in the neooral commissure precisely to avoid a "goldfish mouth" appearance [11]. The authors overlapped the upper and lower parts of the orbicularis oris muscle on the cleft side by approximately 5 $\mathrm{mm}$ when repairing the muscle. If simple approximation is performed between the upper and lower part of the muscle with a horizontal mattress suture, dehiscence of the repaired muscle may occur as the patient moves his or her mouth or makes repetitive facial expressions. The amount of overlapping muscle depends on the degree of cleft. If the degree of the cleft is severe, the amount of overlapping muscle should be larger. However, excessive muscle overlap can cause thickening of the neo-oral commissure. Therefore, we overlapped the orbicularis oris muscle by approximately $5 \mathrm{~mm}$ when repairing the muscle.

Postoperative scarring is one of the most difficult issues that a plastic surgeon must deal with during correction of macrostomia. Because the location of the wound is near the facial expression muscles, such as the zygomaticus major, zygomaticus minor, depressor anguli oris, and risorius muscles, extensive scar migration due to postoperative scarring may cause scar widening or hypertrophic scarring, and even scar contracture in extreme cases. We performed additional scar revision in four patients owing to scar widening. Numerous plastic surgeons have reported various surgical methods for minimizing such scarring $[1,12$ 19], and these methods can be divided largely into skin closure via $\mathrm{Z}$-plasty or W-plasty and simple linear closure. According to surgeons who prefer Z-plasty or W-plasty [12-16], one of the key surgical goals for macrostomia is prevention of inferolateral migration and relapse of the neo-oral commissure due to wound scar contracture. Using Z-plasty or W-plasty can result in less scar contracture than occurs with linear wound scars, and the scar can be minimized by placing the scar parallel to the nasolabial fold and the marionette line. Even if scar contracture occurs, performing Z-plasty can extend the length of the skin and soft tissue, and such an extension in length can be reflected in the contracted scar. Conversely, according to surgeons who prefer simple linear closure $[1,17-19]$, the direction of the wound remaining after dissection of the tissue on the cleft side after surgical design is parallel to the relaxed skin tension line; thus, there is no need to create additional skin scars on the healthy normal tissues. Moreover, the size of the scar that forms after simple linear closure is not much greater than that of the scar following Z- plasty or W-plasty. No reports have yet explored whether distinctly better outcomes are obtained from Z-plasty or W-pasty versus simple linear closure; as such, various surgical techniques continue to be introduced with much debate.

In our study, all the patients were children, and the wound during skin closure on the cleft side was located near the facial expression muscles. Consequently, we performed Z-plasty based on the expectation that doing so would yield more favorable outcomes, considering the likelihood of scar migration due to the extensive movement of the facial expression muscles. In the long-term follow-up of the patients (more than 10 years since surgery), the scars from Z-plasty appeared similar to the surrounding tissues, suggesting no major difference compared to scars from linear wounds. With respect to designing the neooral commissure, performing surgery on the point of symmetry with the normal side caused inferolateral migration of the neooral commissure due to postoperative scar contracture. Therefore, beginning around 1999, surgical procedures for the neooral commissure on the cleft side that also involved Z-plasty were designed with a $1-\mathrm{mm}$ medial overcorrection relative to the normal side. When the 1-mm medial overcorrection was applied, the overcorrected neo-oral commissure on the cleft side migrated slightly in the inferolateral direction, which offset the overcorrection and thereby yielded symmetry with the normal side. Moreover, according to Bae et al. [19], the most favorable outcomes in terms of preventing postoperative relapse of the neo-oral commissure on the cleft side were achieved by designing the neo-oral commissure to be $1 \mathrm{~mm}$ medial, instead of 1 $\mathrm{mm}$ lateral, to the point of symmetry. Further, although efforts have been made to determine the location of the expected point of the neo-oral commissure on the cleft side to facilitate symmetry of both oral commissures, lateral displacement of the neooral commissure occurred in most cases. This is consistent with our opinion that lateral displacement of the neo-oral commissure would occur owing to the significant amount of postoperative facial muscle movement and scar contracture of the skin and soft tissue.

We calculated the symmetry of both sides of the lips on postoperative photos using Photoshop during the follow-up period. To minimize bias, a single evaluator performed all measurements. The mean distance ratio of 1.048 indicates that the cleft and normal sides were symmetrical, and even though medial overcorrection was performed during the operation, both sides of the lips were symmetrical due to inferolateral migration of the neo-oral commissure on the cleft side. However, this study has certain limitations. First, since this procedure was performed to correct a rare congenital anomaly, the number of patients who were evaluated was insufficient. Furthermore, because the photos were 
evaluated in two dimensions, the measured length may have varied depending on the angle at which the picture was taken.

In conclusion, correction of macrostomia in patients with Tessier number 7 cleft should be aimed at restoring lip function via appropriate repair of the orbicularis oris muscle and resolving aesthetic issues via creation of a neo-oral commissure that is symmetrical to the normal side. In doing so, considering the postoperative inferolateral migration of the neo-oral commissure, performing skin closure together with Z-plasty, and performing a 1-mm medial overcorrection may be helpful for achieving symmetry of the lips.

\section{NOTES}

\section{Conflict of interest}

No potential conflict of interest relevant to this article was reported.

\section{Ethical approval}

The study was approved by the Institutional Review Board of Kyungpook National University Chilgok Hospital (IRB No. KNUCH 2018-12-012) and performed in accordance with the principles of the Declaration of Helsinki. Written informed consents were obtained.

\section{Patient consent}

The patients provided written informed consent for the publication and the use of their images.

\section{ORCID}

Jeong Yeop Ryu https://orcid.org/0000-0003-2812-5051

Pil Seon Eo https://orcid.org/0000-0003-1909-7298

Lulu Tian https://orcid.org/0000-0002-3449-8084

Joon Seok Lee https://orcid.org/0000-0002-1580-0487

Jeong Woo Lee https://orcid.org/0000-0003-4903-6066

Kang Young Choi https://orcid.org/0000-0002-7735-1939

Jung Dug Yang https://orcid.org/0000-0002-9121-3604

Ho Yun Chung https://orcid.org/0000-0001-7359-3044

Byung Chae Cho https://orcid.org/0000-0002-9395-8896

\section{REFERENCES}

1. Franco D, Franco T, da Silva Freitas R, et al. Commissuroplasty for macrostomia. J Craniofac Surg 2007; 18:691-4.

2. Fadeyibi IO, Ugburo AO, Fasawe AA, et al. Macrostomia: a study of 15 patients seen in Lagos, Nigeria and proposal for a classification of severity. J Plast Surg Hand Surg 2010;44: 289-95.
3. Sowande OA, Anyanwu LJ, Ademuyiwa AO, et al. Macrostomia: a report of three cases. Ann Plast Surg 2011;66:3546.

4. Gunturu S, Nallamothu R, Kodali RM, et al. Macrostomia: a review of evolution of surgical techniques. Case Rep Dent 2014;2014:471353.

5. Neligan P, Rodriguez ED, Losee JE. Plastic surgery, 4th ed. vol 3. New York: Elsevier Saunders; 2018.

6. Tessier P. Anatomical classification facial, cranio-facial and latero-facial clefts. J Maxillofac Surg 1976;4:69-92.

7. Kobraei EM, Lentz AK, Eberlin KR, et al. Macrostomia: a practical guide for plastic and reconstructive surgeons. J Craniofac Surg 2016;27:118-23.

8. Stark RB, Saunders DE. The first branchial syndrome: the oral-mandibular-auricular syndrome. Plast Reconstr Surg Transplant Bull 1962;29:229-39.

9. Powell WJ, Jenkins HP. Transverse facial clefts: report of three cases. Plast Reconstr Surg 1968;42:454-9.

10. Simonse E, Panis B, Busari JO. Unilateral macrostomia in the newborn: a rare congenital anomaly of the oral commissure. BMJ Case Rep 2016;2016:bcr2016216752.

11. Boo-Chai K. The transverse facial cleft: its repair. Br J Plast Surg 1969;22:119-24.

12. Sugihara H, Ohura T, Ishikawa T. Commissuroplasty for congenital macrostomia. Jpn J Plast Reconstr Surg 1985;28: 404-12.

13. Ono I, Tateshita T. New surgical technique for macrostomia repair with two triangular flaps. Plast Reconstr Surg 2000; 105:688-94.

14. Eguchi T, Asato PH, Takushima A, et al. Surgical repair for congenital macrostomia: vermilion square flap method. Ann Plast Surg 2001;47:629-35.

15. Yu CC, Goh RC, Lo LJ, et al. Surgical repair for macrostomia: significance of Z-plasty limb directions. Ann Plast Surg 2010;64:751-4.

16. Woods RH, Varma S, David DJ. Tessier no. 7 cleft: a new subclassification and management protocol. Plast Reconstr Surg 2008; 122:898-905.

17. Mohan RP, Verma S, Agarwal N, et al. Bilateral macrostomia. BMJ Case Rep 2013;2013:bcr2013010429.

18. Hikosaka M, Nakajima T, Ogata $H$, et al. Refined simple line closure for macrostomia repair: designing a mucosal triangular flap on the commissure region. J Craniomaxillofac Surg 2009;37:341-3.

19. Bae YC, Kang KD, Kim KH. Alteration in surgical technique of Tessier classification number 7 cleft. J Korean Soc Plast Reconstr Surg 2011;38:143-7. 